\title{
Les résultats cosmologiques de la mission Planck
}

\author{
Nabila Aghanim et Hervé Dole (herve.dole@ias.u-psud.fr) \\ Institut d'Astrophysique Spatiale d'Orsay (UMR8617, CNRS et Université Paris-Sud), \\ 91405 Orsay Cedex \\ Membres de la collaboration Planck
}

\section{Le satellite Planck a été lancé}

en 2009 pour mesurer avec

une précision sans précédent

les propriétés du rayonnement

fossile - aussi appelé fond diffus

cosmologique, sorte d'écho

lumineux du Big Bang.

Les scientifiques de la collaboration

Planck ont publié plusieurs séries

de résultats, parmi lesquels

les mesures les plus précises

jamais obtenues de l'âge

et de la composition globale

de I'Univers. De plus, les données

apportent un éclairage nouveau

sur deux périodes particulièrement

énigmatiques : I'inflation juste

après le Big Bang, et la sortie

des « âges sombres » quand

les premières étoiles et galaxies

se sont formées. L'analyse

des données de Planck fournit,

en outre, une mine de

renseignements concernant

la formation des grandes

structures.
L'histoire de l'Univers, telle qu'appréhendée par les cosmologistes, qu'ils soient observateurs, expérimentateurs ou théoriciens, est relativement bien décrite par le "scénario du Big Bang " qui, bien que contenant des variantes et des imperfections, satisfait un grand nombre de tests d'observation. Ce "modèle cosmologique standard " s'est enrichi avec le temps, nourri à la fois de nouvelles données plus précises et de réflexions plus complexes, afin de tenter de répondre à plusieurs questions sur la genèse et l'évolution des structures cosmiques (galaxies, amas de galaxies). Dès lors, les scientifiques cherchent à mettre au défi les différentes variantes du modèle, en effectuant des mesures de nature à les départager et, éventuellement, à exclure certaines de ces variantes. L'une des observables majeures en cosmologie est le rayonnement fossile - ou fond diffus cosmologique, en anglais Cosmic Microwave Background (CMB, acronyme que nous utiliserons dans la suite) -, découvert en 1964 par A. Penzias et R.W. Wilson. Les faibles inhomogénéités $\mathrm{du} \mathrm{CMB}$, i.e. les zones du ciel avec des brillances ou des températures différentes de la moyenne par moins de $0,001 \%$, mises en évidence pour la première fois en 1992 par le satellite COBE de la NASA, sont reliées aussi bien, en aval, aux germes ayant donné naissance aux galaxies que, en amont, aux processus physiques dans l'Univers primordial.

\section{Le fond cosmologique et ses inhomogénéités}

Dans cet Univers primordial très dense et chaud sous la forme d'un plasma, la lumière ne pouvait pas se propager librement : l'Univers était opaque, comme au centre d'une étoile, à cause de la diffusion Thomson - entre lumière (photons) et matière chargée (essentiellement électrons). À ce stade, lumière et matière sont couplées, mais la matière subit des perturbations. La compétition entre la gravité (qui domine la dynamique de croissance des perturbations de matière) et la pression des photons (piégés par leurs interactions avec la matière, et qui s'opposent à l'effondrement gravitationnel) génère des oscillations : des ondes acoustiques (analogues aux ondes sonores), qui se propagent dans le plasma. Sous l'effet conjugué de l'expansion de l'Univers et de la (re)combinaison graduelle entre électrons et protons pour former des atomes d'hydrogène, le libre parcours moyen des photons dans le plasma croît. Lorsque l'Univers devient neutre, environ 375000 ans après le Big Bang (soit un décalage spectral $^{(a)}$ vers le rouge de $1090+/-2,5$ précisément), la matière et les photons se découplent. La lumière est alors libre de se propager jusqu'à nous : c'est le CMB détecté par le satellite Planck. Les ondes acoustiques (et les oscillations de température associées) sont "gelées » et impriment sur le CMB des motifs de tailles et de brillances différentes.

\section{La mission Planck}

Décidé par l'Agence spatiale européenne (ESA) en 1996 (après une longue préparation sous la direction d'une équipe de l'Institut d'astrophysique spatiale (IAS) d'Orsay menée par Jean-Loup Puget, avec plusieurs laboratoires français, le CNES, et une contribution significative de la NASA), le satellite Planck est lancé en mai 2009. C'est presque dix ans après le lancement du satellite américain WMAP (pourtant décidé la même année que Planck), parce 
a
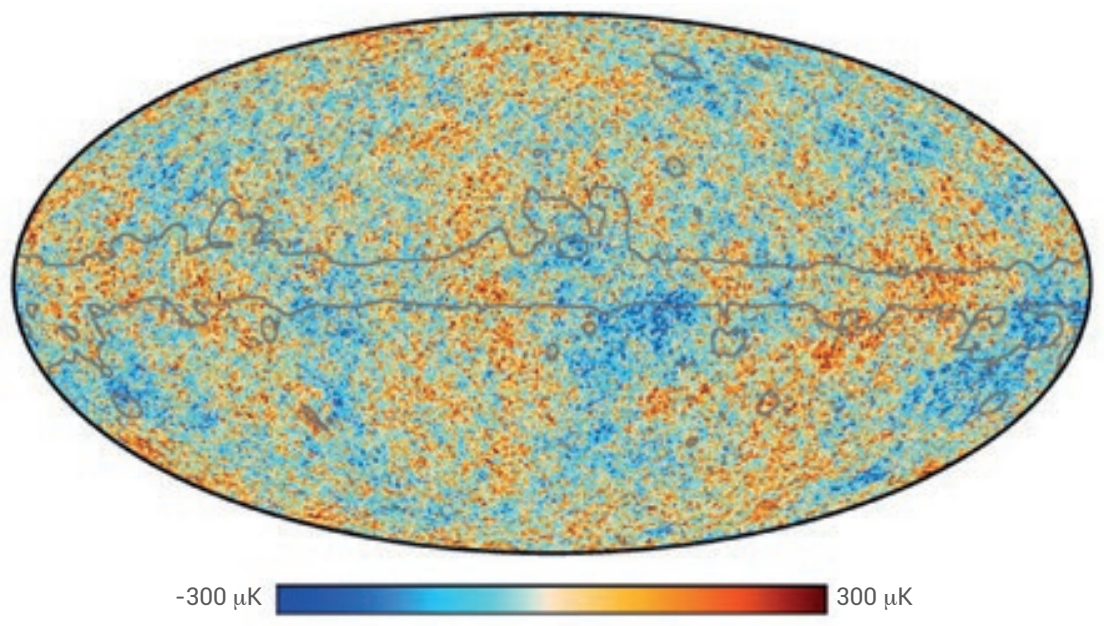

que les stratégies technologiques étaient différentes. En effet, les Européens ont choisi une voie plus ambitieuse, donc plus couteuse, longue et risquée, mais avec à la clef des mesures et des résultats insurpassables pour longtemps : la nouvelle technologie se base sur des détecteurs ultrasensibles - les bolomètres - refroidis à une température record de 0,1 kelvin. Ainsi, une seule année d'observation de Planck équivaut à environ 1000 années d'observation de WMAP. Planck dispose de deux instruments : HFI (High Frequency Instrument) réalisé sous la maitrise d'œuvre de l'IAS à Orsay et sous la responsabilité de J.L. Puget, et LFI (Low Frequency Instrument) réalisé sous responsabilité italienne. Le satellite Planck a été pensé pour cartographier la totalité du ciel dans le domaine spectral millimétrique $(0,3$ à $11 \mathrm{~mm})$, avec une résolution angulaire relativement fine par rapport à la granularité du CMB. Planck observe non seulement l'intensité mais également la polarisation du CMB et du rayonnement de notre Galaxie, avec une stratégie d'observation permettant de caractériser avec précision et de contrôler les effets instrumentaux pouvant dégrader ou impacter le signal. Pour finir, Planck a une stratégie d'analyse (incluant un contrôle par simulation), afin d'extraire le signal du fond diffus cosmologique des nombreux autres signaux astrophysiques.

Initialement, douze mois d'observations étaient prévus pour cartographier deux fois la totalité du ciel (soit deux "relevés"). Planck-HFI a finalement observé durant 29 mois et a réalisé cinq relevés complets, grâce à la très grande stabilité de son système de refroidissement. Pour la première fois en cosmologie, en raison de la sensibilité exceptionnelle des détecteurs de Planck, la mesure des fluctuations du CMB n'est

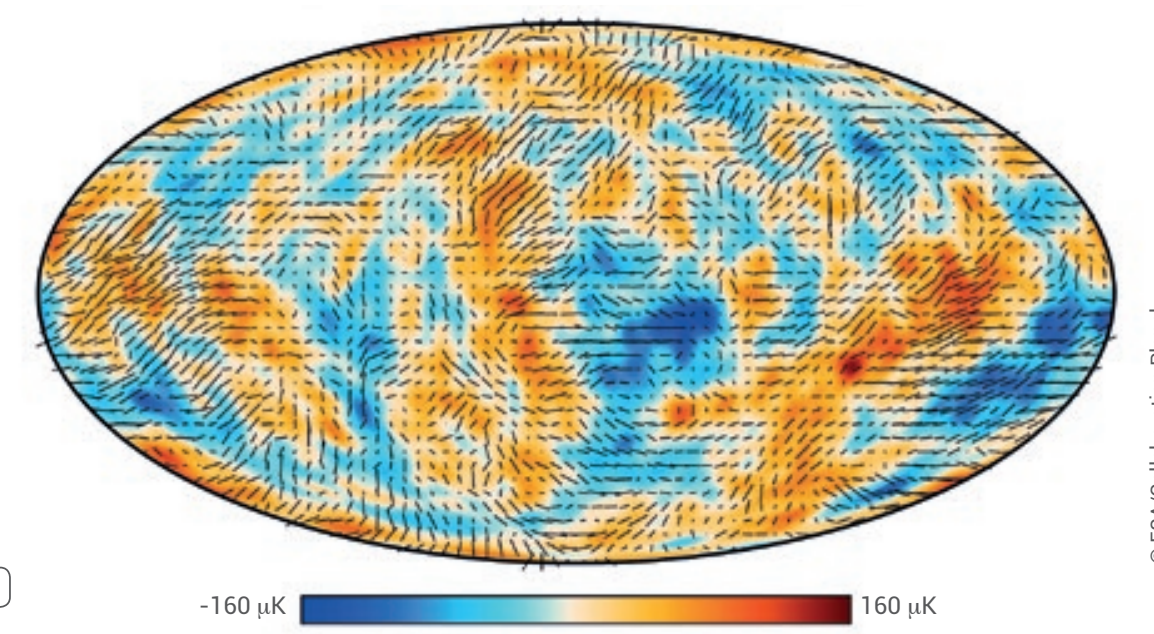

1. Cartes du fond cosmologique observé par le satellite Planck, après soustraction des avant-plans, dont la contribution de la Voie lactée (1,6\% de la surface du ciel). (Figures tirées de "Planck 2018 results. I. Overview, and the cosmological legacy of Planck", accepté pour publication dans A\&A).

(a) Anisotropies de l'intensité (température) du CMB, à la résolution maximale (5 minutes) et avec une sensibilité de quelques $\mu \mathrm{K}$. Les lignes grises délimitent les régions où l'émission d'avant-plan est substantielle. La couleur rouge correspond aux zones plus chaudes et la couleur bleue aux zones plus froides.

(b) Champ de polarisation du CMB, indiqué par des traits noirs de longueur variable, superposé à la carte de température (couleurs) lissée à une résolution réduite à 5 degrés.

plus limitée par cette sensibilité, mais par notre capacité à bien séparer le fond cosmologique des émissions contaminantes d'avant-plan comme celles provenant de la lumière de notre Galaxie, des autres galaxies et des amas de galaxies. La qualité de cette "séparation des composantes astrophysiques " dépend non seulement de la qualité de mesure du CMB lui-même, mais aussi de celle des dits avant-plans. Les observations de Planck à neuf bandes de fréquences différentes, centrées de $30 \mathrm{GHz}$ (longueur d'onde $\lambda \approx 10 \mathrm{~mm}$ ) dans le domaine des micro-ondes jusqu'à $857 \mathrm{GHz}(\boldsymbol{\lambda} \approx 0,3 \mathrm{~mm})$ dans l'infrarouge lointain, assurent une mesure fine des émissions non cosmologiques d'avantplan, en vue de les soustraire du signal total.
Depuis 2010, la collaboration Planck analyse les données et publie les résultats associés dans la revue Astronomy and Astrophysics. La version intégrale et définitive de ces résultats a été publiée le 17 juillet 2018. Les données de Planck révèlent le CMB comme jamais vu auparavant, avec de bien meilleures sensibilité et résolution angulaire. L'image des inhomogénéités de température du CMB (fig. 1a) montre qu'elles ont une distribution en taille dominée par des motifs de l'ordre du degré sur le ciel. Ce sont les motifs associés à la première harmonique des ondes acoustiques, c'est-à-dire le premier pic acoustique. Mais surtout, Planck donne pour la première fois accès à la polarisation ${ }^{(b)}$ fine du CMB sur tout le ciel (fig. 1b). 


\section{Du fond diffus aux paramètres cosmologiques}

Les cosmologistes analysent les images du $\mathrm{CMB}$ avec un outil statistique privilégié - le spectre de puissance angulaire ${ }^{(c)}-$ qui fait office de mesure de corrélation à deux points dans toutes les directions. Dès lors, les spectres de puissance, données et modèles théoriques, sont directement comparables.

Grâce à son excellente résolution angulaire, Planck mesure dix-neuf harmoniques ou pics acoustiques des spectres de puissance des fluctuations de température (fig. 2) et des fluctuations de polarisation E (fig. 3) $\mathrm{du} C \mathrm{CMB}$ : une grande première.

L'importance que revêt cette mesure tient au fait que l'analyse du spectre de puissance du CMB permet de déterminer les paramètres " cosmologiques " qui décrivent le contenu de l'Univers en matière ordinaire et sombre, en neutrinos, en énergie sombre, notamment. Par exemple, la brillance des motifs de taille un degré (l'amplitude du premier pic acoustique) et leur taille angulaire dépendent respectivement de la densité totale d'énergie et de la courbure de l'Univers. Ou encore, la brillance relative des motifs de taille inférieure à un degré (amplitude relative des premier et deuxième pics) dépend de la quantité de matière ordinaire dans l'Univers. Dès lors, plus le spectre de puissance possède de pics acoustiques plus il est «complet » et meilleure sera la précision de la mesure des paramètres cosmologiques. Ces derniers nous révèlent l'ensemble des ingrédients cosmiques, exprimés en terme de leur rapport à la densité totale d'énergie dans l'Univers et notés $\mathrm{W}_{\mathrm{b}}, \mathrm{W}_{\mathrm{CDM}}, \mathrm{W}_{\mathrm{n}}, \mathrm{W}_{\mathrm{L}}$, les indices faisant référence à la matière baryonique (b), à la matière sombre (CDM pour cold dark matter), aux neutrinos (n) ou encore à l'énergie sombre (L). Les paramètres cosmologiques nous renseignent aussi sur les propriétés de l'Univers : son âge, à travers la constante de Hubble $\mathrm{H}_{0}$ qui mesure le rythme d'expansion de l'Univers, et sa géométrie, via son paramètre de courbure $\mathrm{W}_{\mathrm{k}}$, par exemple.

Les spectres de puissance de Planck sont comparés à des milliers de modèles reproduisant, en plus du CMB, tous les signaux mesurés par Planck comme les effets des amas de galaxies, des galaxies, de l'émission de notre Galaxie. On recherche alors l'ensemble des paramètres (cosmologiques, astrophysiques, instrumentaux) qui rendent

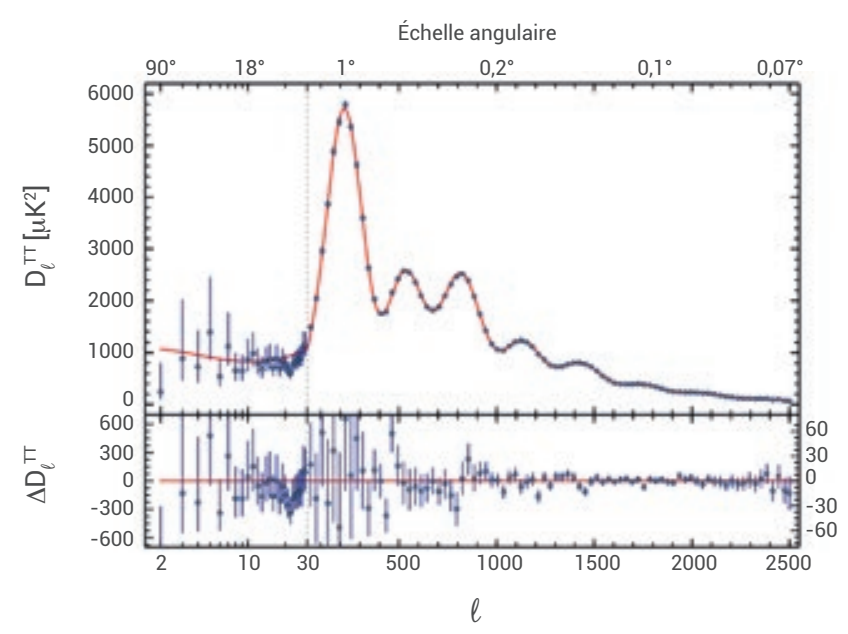

2. Spectre de puissance des fluctuations de température (en microkelvins carrés) du fond diffus cosmologique en fonction de l'échelle angulaire. Les moments multipolaires $\ell$ correspondant aux diverses échelles angulaires sont indiqués le long de l'axe des abscisses.

En haut du graphe : les données de Planck (points et barres d'erreur en bleu) et le meilleur modèle (courbe rouge).

Insert du bas : résidus par rapport au modèle. Noter que le spectre de puissance est construit différemment pour le régime bas $\ell(2<\ell<29)$ et le régime haut $\ell(\ell>29)$.

(Figure tirée de Planck Collab. XIII, A\&A 593 (2015) A13.)

a

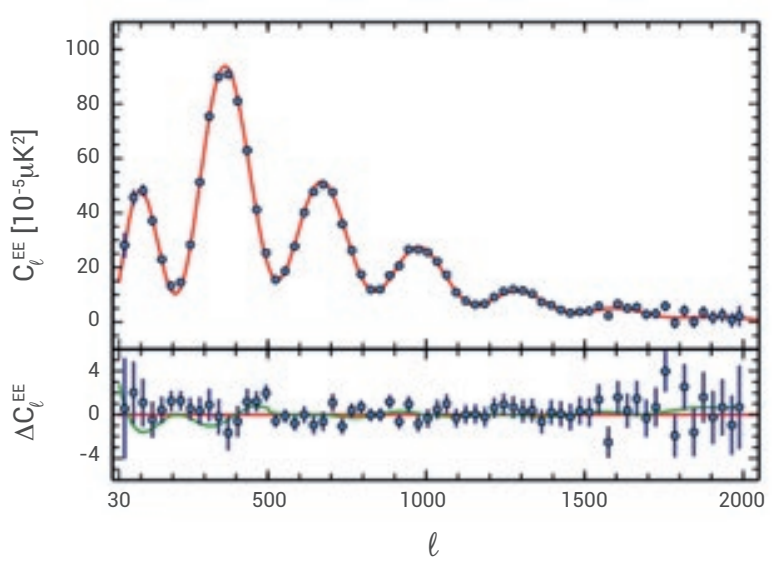

(b)

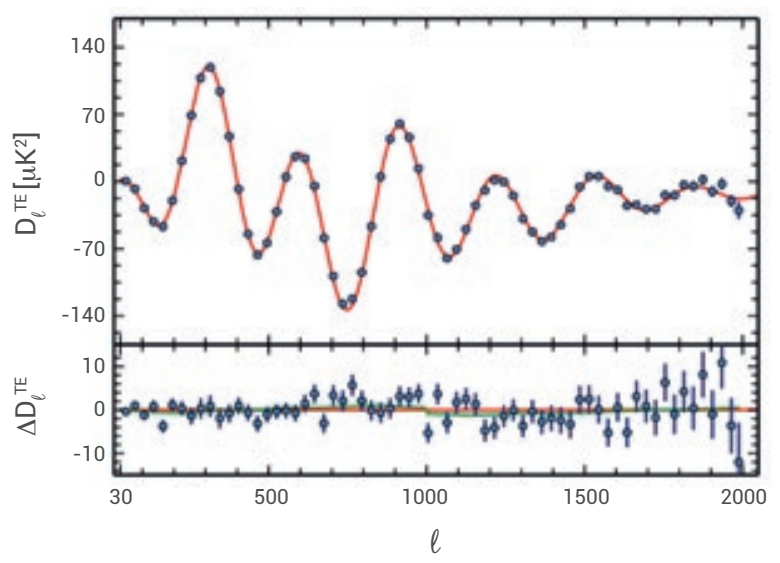

3. Spectres de puissance du fond diffus cosmologique (a) en polarisation E (noté EE) et (b) en corrélation croisée température et polarisation $E$ (noté TE). Mêmes notations que la figure 2. (Figure tirée de Planck Collab. XIII, A\&A 593 (2015) A13.) 
le mieux compte du modèle complet du ciel, étant données les observations. Seuls sont retenus les modèles, et par conséquent l'ensemble des paramètres, qui s'accordent au plus près avec les observations et leurs incertitudes.

Grâce à la qualité inégalée des données de Planck, des mesures d'une précision jamais atteinte ont été obtenues pour les paramètres cosmologiques. Planck a révisé la « composition » de l'Univers. La quantité de matière ordinaire et de matière sombre doit être augmentée d'environ 10\% par rapport aux précédentes estimations. La quantité d'énergie sombre, quant à elle, doit être diminuée d'autant. Le plus remarquable dans les plus récents résultats de Planck est la précision extrême des mesures des paramètres, de l'ordre de 0,6 et $1,1 \%$ pour les densités de baryons et de matière sombre respectivement. Planck mesure un paramètre de courbure de l'Univers de l'ordre de - 0,004 et établit donc que l'Univers est plat et que notre espace a une géométrie euclidienne. Les derniers résultats de Planck utilisant la polarisation du CMB confirment que la constante de Hubble mesurée, $67,27 \mathrm{~km} / \mathrm{s} / \mathrm{Mpc}$, est environ $10 \%$ plus faible que les précédentes estimations. La qualité des données de Planck, et l'accès aux nombreux pics acoustiques dans le spectre de puissance, ont permis pour la première fois de détecter l'effet des neutrinos primordiaux, "émis " lorsque l'Univers était âgé d'une seconde, sur la carte du rayonnement fossile. Cet effet est en accord avec le scénario du Big Bang et le modèle standard de la physique des particules. Planck montre bien que le nombre d'espèces de neutrinos est de trois, tel que prédit par la théorie.

Avec cet ensemble de mesures qui utilisent les spectres de puissance en intensité/ température et en polarisation, il suffit de six paramètres cosmologiques ${ }^{(d)}$ pour en ajuster parfaitement les dix-neuf pics. L'un des résultats majeurs de Planck est donc que le modèle cosmologique "standard " le plus simple (appelé aussi "modèle de concordance ") est favorisé par les données et qu'aucune remise en cause profonde n'est envisageable, même si quelques tensions existent.

\section{L'inflation cosmique}

L'analyse statistique du CMB permet de mieux cerner le processus d'inflation cosmique $^{(e)}$ et donc l'état de l'Univers une fraction infime de seconde après le Big Bang.

Les mesures de Planck renforcent considérablement le modèle de l'inflation dans son expression la plus simple et standard. La mesure d'une très faible valeur du paramètre de courbure de l'Univers $(-0,004)$ indique que l'hypothèse de sa "platitude ", conséquence de l'inflation, est confirmée. Par ailleurs, et pour la première fois, Planck a permis la mesure non ambigüe de l'indice spectral scalaire $^{(\mathrm{f})} \mathrm{n}_{\mathrm{s}}=-0,9645$, avec une précision telle que l'écart à la valeur -1 (caractérisant le cas indépendance de la taille physique) est indiscutable. Cette mesure est conforme aux prédictions de l'inflation, où l'indice $n_{s}$ doit être compris entre - 0,96 et 0,97 . D'autre part, le caractère gaussien de la distribution des inhomogénéités de température du CMB, stipulé par les modèles les plus simples et usités d'inflation standard, est maintenant établi avec la plus grande précision jamais obtenue, rejetant ainsi toute une famille de modèles d'inflation. De plus, grâce à la mesure la plus précise obtenue à ce jour de la polarisation du CMB aux échelles angulaires inférieures au degré $(l>100)$ sur tout le ciel, Planck établit définitivement la nature adiabatique des perturbations de matière, c'est-à-dire que les perturbations de densité sont en équilibre thermique les unes avec les autres.

Une avancée majeure pour conforter de manière déterminante le paradigme de l'inflation standard serait la mesure directe de la polarisation, dite de mode $\mathrm{B}^{(\mathrm{b})}$, aux échelles angulaires supérieures au degré. Cette polarisation est la signature claire des ondes gravitationnelles primordiales générées par la phase d'inflation cosmique. Sa mesure représente la nouvelle limite posée aux expériences visant à mesurer le CMB depuis le sol ou dans l'espace. L'importance que revêt la confirmation " ultime » d'une phase d'inflation cosmique par la mesure des modes B de polarisation impose une approche très sérieuse et prudente du traitement des données, en particulier de la séparation du signal cosmologique de celui associé à notre Galaxie qui domine aux grandes échelles angulaires. Ce type d'analyse poussée a été réalisé avec les données de Planck combinées à celles du télescope au sol BICEP2/KECK. Elle a mis en évidence que la part d'émission due à la poussière galactique avait été significativement sous-estimée, ce qui avait conduit l'équipe BICEP à une interprétation erronée de leur signal comme étant un signal cosmologique. Aujourd'hui, l'analyse des données de Planck seules et celles les combinant aux données de BICEP2/KECK indiquent que le paramètre mesurant l'importance du signal produit par les ondes gravitationnelles primordiales, correspond à une valeur supérieure - pas encore à une détection. La quête de la trace des ondes gravitationnelles primordiales dans la polarisation du CMB ne fait que commencer.

\section{La reionisation de l'Univers}

Une autre période énigmatique de l'histoire de l'Univers est la « reionisation » qui désigne l'époque, quelques centaines de millions d'années après la recombinaison, lors de laquelle les atomes d'hydrogène sont de nouveau séparés en électrons et protons. La reionisation est communément associée à l'émission de photons ionisants par les premiers objets lumineux (première génération d'étoiles, premières galaxies), même si d'autres hypothèses sont avancées comme la production de photons énergétiques et ionisants lors de la désintégration de particules de matière sombre.

Les observations du CMB apportent un éclairage unique et original sur la reionisation. En effet, celle-ci laisse une empreinte très caractéristique dans le CMB sous la forme d'un pic dans le spectre de puissance de la polarisation de type $\mathrm{E}$ aux très grandes échelles angulaires (fig. 3). Seules des observations de l'ensemble de la voute céleste permettent d'accéder à ce signal. Par ailleurs et de nouveau, la séparation de l'émission polarisée de notre Galaxie est déterminante pour isoler le signal associé à la reionisation. En 2016 et grâce à une analyse particulièrement longue et détaillée de tous les effets instrumentaux intervenant aux grandes échelles angulaires, la collaboration Planck a pu mesurer l'opacité t de l'Univers dû à la reionisation. La valeur obtenue $(t=0,055 \pm 0,009)$ permet de déduire que la reionisation s'est produite à un décalage vers le rouge entre 8 et 9. Les données de Planck indiquent aussi que le processus de reionisation n'a pas été très efficace avant un décalage spectral de 15. Ceci pourrait suggérer que les premières sources lumineuses qui se seraient formées 
$\gg>$

avant un décalage de 15 n'auraient pas pu libérer efficacement des photons ionisants. Ces nouvelles mesures de Planck semblent confirmer les observations de galaxies très lointaines par le télescope spatial Hubble, dont on pense qu'elles sont responsables de l'émission de grandes quantités de photons ionisants.

\section{Les grandes structures}

Les perturbations initiales de densité générées aux premiers instants de l'Univers accrètent de la matière sous l'effet de la gravité et croissent. Elles continuent de grandir par fusions successives et s'organisent sous la forme d'un réseau de matière baryonique et de matière sombre. Lorsqu'elles sont assez denses, elles forment en leur sein les premières étoiles et donnent naissance aux toutes premières galaxies, qui évoluent ensuite sous la forme de structures cosmiques complexes.

Il existe deux méthodes pour visualiser le réseau de filaments de matière sombre. $\mathrm{La}$ première consiste à observer comment les galaxies se distribuent dans l'espace (et dans le temps). La seconde consiste à identifier comment la gravité, induite par la matière sombre dans les filaments, courbe les rayons lumineux issus des galaxies distantes et distord leurs images. C'est l'effet de lentille gravitationnelle faible. Cet effet intervient aussi en distordant et lissant les motifs les plus petits (quelques minutes d'arc sur le ciel) de l'image du CMB utilisée comme lumière d'arrière-plan. Ces distorsions sont employées dans l'analyse fine des cartes du CMB effectuée par la collaboration Planck pour reconstituer la distribution sous-jacente de matière sombre. Grâce à l'effet de lentille gravitationnelle faible, Planck a permis pour la première fois de visualiser l'accumulation (sur la ligne de visée) de matière sombre sur la totalité du ciel (fig. 4), et en a ainsi fourni une cartographie complète et une caractérisation de ses propriétés statistiques.

Cette carte représente en quelque sorte l'ensemble des écrins de matière sombre dans lesquels les galaxies vont se former et évoluer. On les désigne sous la terminologie de "puits de potentiel de matière sombre ». Le gaz des amas de galaxies va aussi s'y agréger, pour y atteindre des températures extrêmes jusqu'à des centaines de millions de degrés.

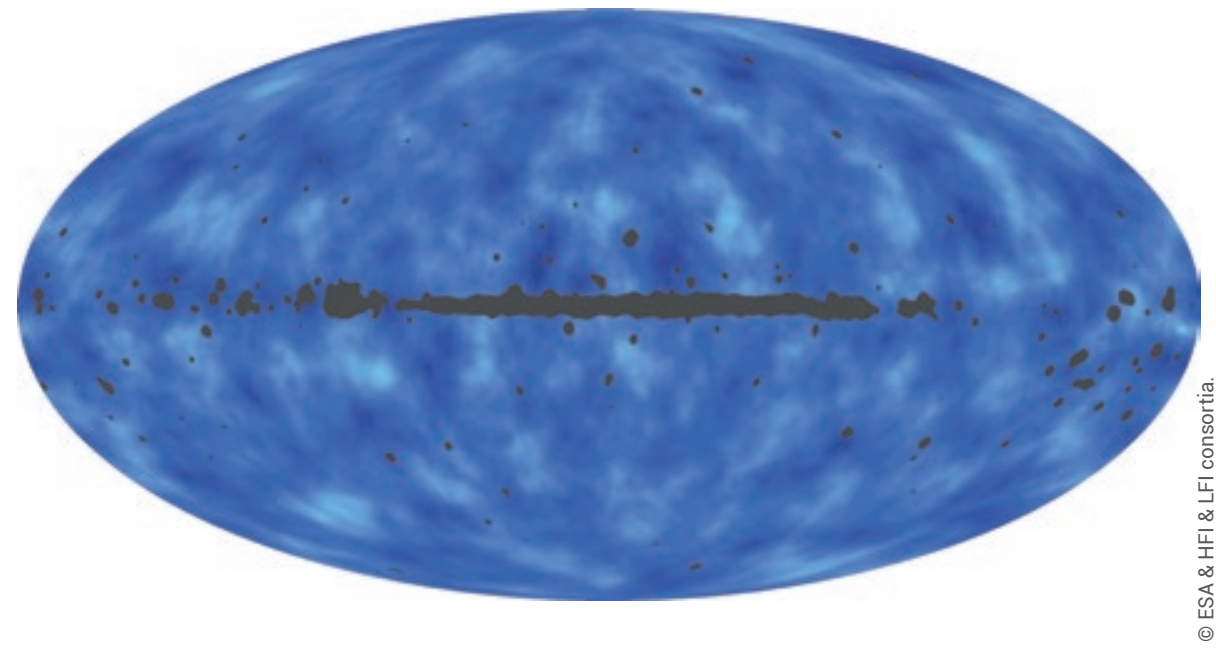

4. Carte du potentiel gravitationnel obtenu par l'analyse de l'effet de lentille gravitationnelle faible sur la carte du fond diffus cosmologique. Ce potentiel est relié à la présence de matière sombre projetée sur la ligne de visée. Les régions avec plus de masse sont en couleur claire, celles avec moins de masse en foncé. (Figure tirée de Planck Collab. I, A\&A 593 (2015) A1.)

$545 \mathrm{GHz}$

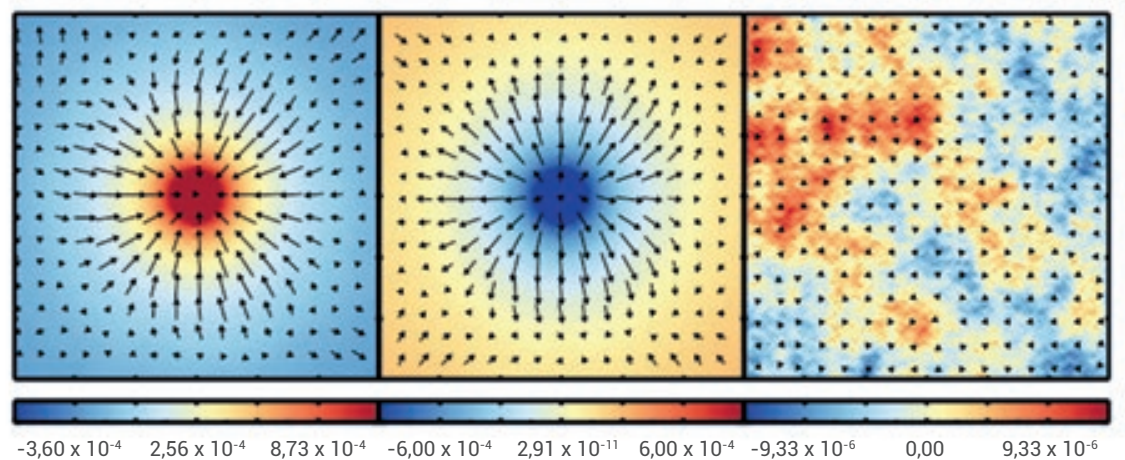

5. Visualisation dans l'espace réel de la corrélation entre le fond diffus infrarouge (FDI) de galaxies et le potentiel gravitationnel. Extraits de un degré carré de la carte des températures du fond diffus infrarouge mesurée à la fréquence $545 \mathrm{GHz}$ de Planck-HFI, réalisés en superposant les zones centrées sur les 20000 pics d'intensité les plus brillants (à gauche), les moins brillants (au milieu) ou choisies aléatoirement (à droite, pour s'assurer que la méthode n'induit aucun effet systématique). Les couleurs traduisent l'intensité de l'émission du fond diffus infrarouge, les flèches tracent par leur longueur et leur direction la déflexion par effet de lentille gravitationnelle sur le rayonnement fossile. On voit que les maxima de température du FDI (en rouge) défléchissent la lumière vers l'intérieur et correspondent donc à des puits de potentiel gravitationnels, alors que les minima de température (en bleu) correspondent à des maxima de potentiel. (Figure tirée de Planck Collab. XVIII, A\&A 571 (2013) A18.)

En corrélant la carte de matière sombre déduite de l'effet de lentille gravitationnelle sur le CMB aux positions des galaxies et aux amas connus, la collaboration Planck a montré de manière magistrale que le modèle hiérarchique de formation des structures cosmiques décrit dans ses grandes lignes parfaitement bien la distribution et l'agrégation des objets astronomiques jusqu'à des époques très reculées, correspondant à un décalage vers le rouge de l'ordre de 2 .
Par ailleurs, la corrélation des cartes de l'effet de lentille gravitationnelle et de l'émission du FDI (fond diffus infrarouge, qui représente l'ensemble de l'émission des galaxies de l'Univers le long de son histoire et trace la formation stellaire dans les galaxies), toutes deux mesurées par Planck, illustre parfaitement la concomitance de la formation des étoiles dans les galaxies avec la présence d'une densité de matière sombre distordant le CMB (fig. 5). 
Enfin, l'analyse des images du FDI révèle des objets inconnus qui apparaissent « froids " dans le jargon des astrophysiciens, c'est-àdire présentent une émission spectrale très décalée vers le rouge. Ces "points froids du FDI » pourraient être des proto-amas de galaxies (le chainon manquant dans la formation des structures) ou des galaxies individuelles très fortement amplifiées par effet de lentille gravitationnelle. Dans les deux cas, ces objets se situeraient à des décalages spectraux supérieurs à 2 . Un grand ensemble d'observations, notamment de l'observatoire européen spatial Herschel, a permis de prouver qu'en effet, sur des centaines d'objets "froids ", certains se situent bien à des redshifts supérieurs à 2 (fig. 6).

La qualité des données de Planck nous permet donc de sonder aussi la formation des toutes premières structures individuelles et de compléter la "généalogie " des galaxies et des amas.

\section{Les amas de galaxies}

Les galaxies se regroupent pour former des amas à l'intersection des filaments du réseau cosmique. La matière ordinaire sous forme d'un gaz est chauffée à des températures de l'ordre de dizaines à la centaine de millions de degrés dans les puits de potentiel de matière sombre. Les photons du CMB interagissent avec ce gaz chaud. Ils acquièrent un peu plus d'énergie à la sortie des amas qu'à leur entrée - un effet dénommé "Sunyaev-Zel'dovich " (SZ). Grâce à cette manifestation spectrale unique et caractéristique (voir l'encadré, p. 10), Planck est particulièrement adapté pour la découverte des amas de galaxies. Une combinaison de techniques adaptées a permis à la collaboration Planck de mettre en évidence plus d'un millier d'amas de galaxies (dont plus de 500 nouveaux amas), ainsi que des super-amas. Environ vingt ans après le premier catalogue d'amas complet dans le domaine des rayons $\mathrm{X}-$ produit par le satellite ROSAT - les catalogues établis par Planck dans les domaines millimétrique et submillimétrique ont révélé une population d'amas massifs et lointains jamais observés auparavant.
L'observation de ces amas permet de mieux comprendre leur physique, mais aussi de mesurer certains paramètres cosmologiques, puisque la formation de ces objets massifs y est très sensible. Avec un souséchantillon de plusieurs centaines de ces amas, la collaboration Planck a obtenu les mesures les plus précises de ces paramètres, notamment la densité de matière et l'amplitude des perturbations initiales. De plus, pour la première fois, la mesure de l'effet SZ intégrée sur toute la sphère céleste, effectuée par Planck, a mis en évidence la contribution des amas non résolus. Avec ce signal intégré de l'effet SZ, les paramètres cosmologiques ont été contraints.

Cependant, les résultats obtenus avec les mesures SZ ne sont pas tout à fait en accord avec les paramètres cosmologiques issus de la mesure du CMB par Planck, en particulier l'amplitude des perturbations initiales $\mathrm{S}_{8}$. Cet écart n'est pas assez important pour remettre en cause fondamentalement le modèle, mais il est suffisamment grand pour provoquer un débat dans la communauté, car il ouvre la possibilité d'une mesure indirecte de la masse des neutrinos, ou d'une révision des estimations des masses des amas de galaxies.
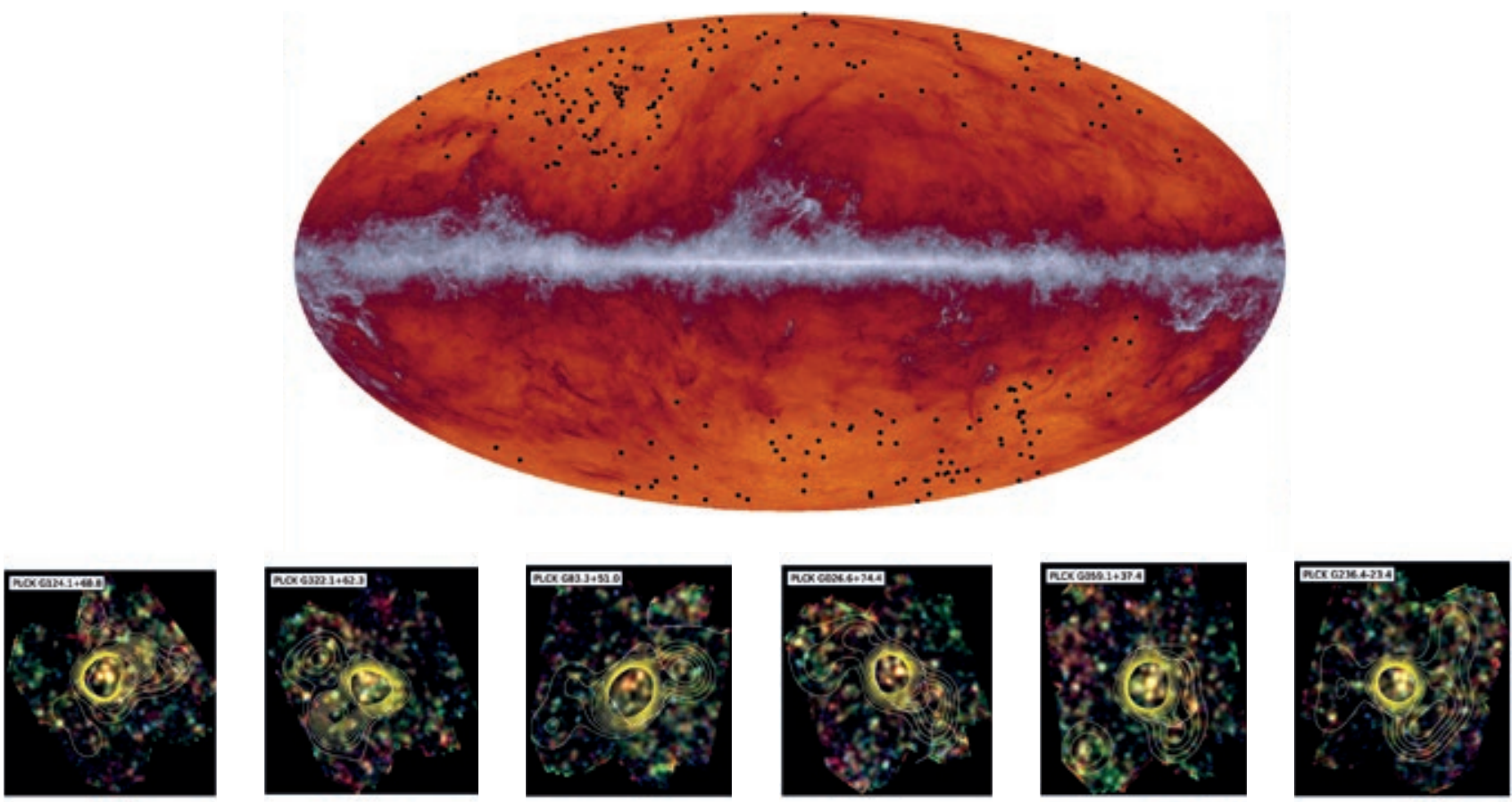

6. Les points froids du fond diffus cosmologique, observés par les observatoires Planck et Herschel. En haut : la totalité du ciel observé par Planck, à la fréquence de $545 \mathrm{GHz}$ (soit environ $550 \mu \mathrm{m}$ de longueur d'onde). La bande blanche horizontale correspond aux poussières de la Voie lactée. Les points noirs indiquent la position des candidats observés ultérieurement par Herschel. En bas, les images dans les directions de six de ces points froids, prises par l'instrument SPIRE de Herschel à 250, 350 et $500 \mu \mathrm{m}$ de longueur d'onde, avec les contours de densité surfacique des galaxies observées. (Figure tirée de Planck Collab., Planck intermediate results, XXVII, A\&A 582 (2015) 30). 


\section{Conclusion}

Grâce à sa cartographie complète du ciel à neuf longueurs d'onde et avec une résolution angulaire de 4,5 minutes d'arc, Planck, le satellite européen dédié à l'étude $\mathrm{du}$ fond diffus cosmologique, a ouvert une fenêtre d'observation complètement nouvelle entre 100 et $900 \mathrm{GHz}$ (de 3 millimètres à 350 microns de longueur d'onde). Planck a permis de faire des avancées significatives dans de nombreux domaines de l'astrophysique et de la cosmologie. Il a révisé la détermination des paramètres cosmologiques, comme la composition ou l'âge de l'Univers. Il a découvert des centaines de nouveaux amas, superamas et proto-amas de galaxies, et des pouponnières d'étoiles. Finalement, Planck a permis d'établir solidement le paradigme de l'inflation, de sonder la reionisation et de vérifier la validité du scénario hiérarchique de formation des structures cosmiques.

\section{Références}

- Articles scientifiques de la collaboration Planck : www.cosmos.esa.int/web/ planck/publications

- Site grand public en français, avec images et vidéos : www.planck.fr

- Productions scientifiques de la collaboration Planck : www.cosmos.esa.int/web/planck

\section{L'effet Sunyaev-Zel'dovich}

L'effet Sunyaev-Zel'dovich (SZ) provient de l'interaction (Compton inverse) entre les photons issus du fond cosmologique avec le gaz chaud présent à l'intérieur des amas de galaxies, provoquant de légers changements de fréquence des photons. L'effet SZ est très caractéristique car, observé aux fréquences inférieures à $217 \mathrm{GHz}$ (soit 1,3 mm de longueur d'onde), l'amas de galaxies apparait comme une zone donnée du ciel plus sombre que la moyenne (signal négatif), alors qu'il est plus brillant (signal positif) aux plus hautes fréquences (fig. E1). L'effet est nul à la fréquence $217 \mathrm{GHz}$, il n'y a alors pas de variation de brillance.

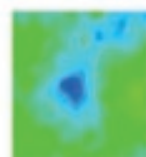

$44 \mathrm{GHz}$

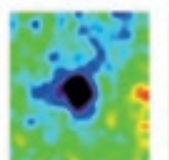

$70 \mathrm{GHz}$

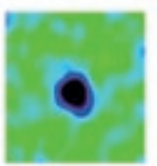

$100 \mathrm{GHz}$

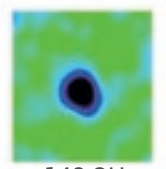

$143 \mathrm{GHz}$

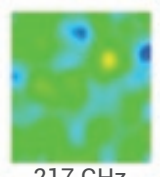

$217 \mathrm{GHz}$

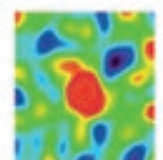

$353 \mathrm{GHz}$

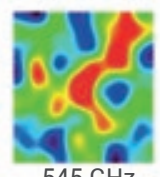

$545 \mathrm{GHz}$ a

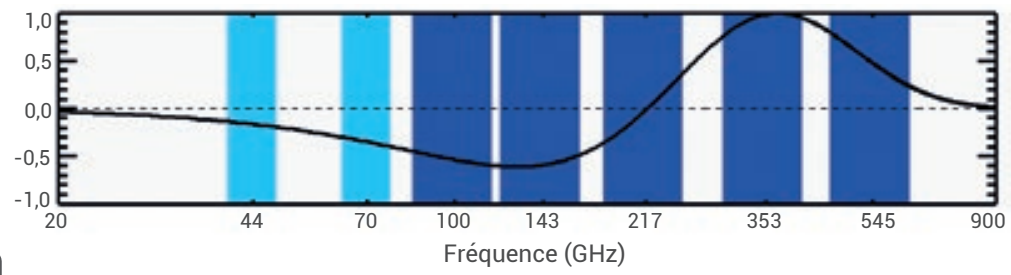

E1. (a) La frise d'images illustre l'effet SZ dans le cas d'un amas de galaxies connu et proche, Abell 2319. La variation de couleur due à cet effet est observée avec les deux instruments de Planck, LFI et HFI, de 44 à $545 \mathrm{GHz}$. Le signal négatif (zone sombre centrale) à basse fréquence est indiqué en bleu, alors que le signal positif (zone brillante centrale) à haute fréquence est indiqué en rouge.

(b) Le schéma en bas de la figure montre la perte ou le gain en énergie, d'un point de vue théorique, dus à l'effet SZ (bandes de sensibilité de LFI à 44 et $70 \mathrm{GHz}$ en bleu clair, bandes de sensibilité de HFI de 100 à $545 \mathrm{GHz}$ en bleu foncé). L'accord entre prédiction et observation est remarquable. 